\title{
Automatische visuelle Datensammlung aus Materialflusssystemen und ihre Anwendung in Simulationsmodellen
}

\author{
Automatic visual data collection in material flow systems \\ and the application to simulation models
}

\author{
Gábor Bohács \\ Lehrstuhl für Baumaschinen, Fördertechnik und Betriebslogistik, \\ Fakultät Transport- und Ingenieurwesen, \\ Budapest University of Technology and Economics \\ Kai F. Semrau \\ Lehrstuhl für Förder- und Lagerwesen, Fakultät Maschinenbau, \\ Technische Universität Dortmund
}

\begin{abstract}
imulation ist heute eine unentbehrliche Komponente $\checkmark$ bei der Planung und Analyse von modernen Materialflusssystemen. Ihr Nutzen hängt in hohem Maße davon ab, wie akkurat sie das physikalische System modellieren kann. In diesem Artikel wird ein Kamerabasiertes System vorgestellt, welches in bestimmten Einsatzfällen bei der Datensammlung behilflich sein kann. Vor- und Nachteile des Systems werden diskutiert und die Einsatzfälle abgegrenzt. Am Ende des Artikels wird das Kamera-basierte Datensammlungssystem an Beispielen verdeutlicht und es wird gezeigt, wie die Ergebnisse in Simulationsmodellen angewendet werden können.
\end{abstract}

[Schlüsselwörter: visuelle Datensammlung, Simulation, Simulationsmodell, Materialflusssystem]

$\mathbf{T}$ oday simulation is an essential element during planning and analyzing processes of material flow systems. Usability depends on modeling capability of the physical system. This paper describes a camera-based system, which enables data collection in different use cases. Systems advantages and disadvantages will be discussed and the use cases will be differentiated. At the end of the paper the camera-based data collection system will be illustrated with some examples and it will be demonstrated, how results can be integrated in simulation models.

[Keywords: Visual data collection, simulation, modeling, material handling system]

\section{EINFÜHRUNG}

Simulation ist heute eine unentbehrliche Komponente bei der Planung und Analyse von modernen Materialflusssystemen. Ihre Nutzbarkeit hängt in hohem Maße davon ab, wie gut sie das physikalische System nachbilden kann. Bei der Anwendung von Simulationen wird zwischen online- und offline-Modellen unterschieden. Die meisten eingesetzten Modelle werden offline verwendet. Die Eingangsdaten können in diesem Fall sowohl aus dem tatsächlichen Betrieb als auch aus der Planungstätigkeit gewonnen werden. Da die Dateneingabe nur einmalig notwendig ist, besitzen diese Modelle die nachteilige Eigenschaft, dass der Unterschied zwischen den Modellergebnissen und dem physikalischen System mit der Zeit größer wird. Abb.1 zeigt diese Eigenschaft für ein beispielhaftes logistisches Merkmal. Online-Simulationen besitzen dagegen einen wesentlich kleineren Modellfehler. Daher werden diese für die Unterstützung von kurzfristigen Entscheidungen (z. B.: Wochenpläne) angewendet. Ein kleinerer Modellfehler kann aber nur mit Hilfe von genauen Modellen erreicht werden, was die Anwendung sehr detaillierter Betriebsdaten erfordert. Ein weiterer wichtiger Punkt ist die ständige Aktualisierung oder auch Adaptierung - des Modells. Diese benötigt solche Systeme und Methoden, die zur Beobachtung und Datenerfassung aus dem Materialflusssystem dienen. In diesem Artikel wird ein Kamera-basiertes System vorgestellt, welches in bestimmten Einsatzfällen bei der Datensammlung behilflich sein kann. Durch Beispiele wird verdeutlicht, wie die Ergebnisse in Simulationsmodellen angewendet werden können. 


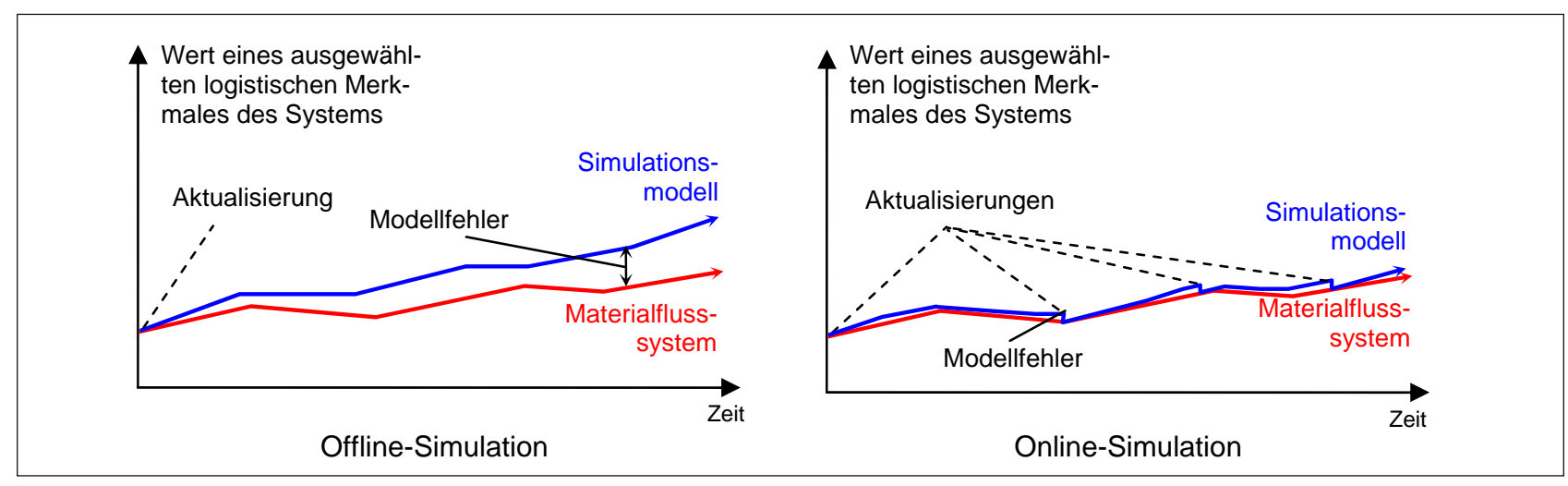

Abbildung 1: Vergleich von Simulationsmodell-Grundtypen

\section{ANWENDBARKEIT EINES KAMERASYSTEMS FÜR DIE DATENSAMMLUNG}

In der Praxis gibt es verschiedene Methoden, um Materialflussdaten für Simulationszwecke zu gewinnen. Die anwendbaren Methoden können in die folgenden Gruppen unterteilt werden [Pre95]:

- Manuelle Datenaufnahme durch an dem Prozess teilnehmende oder unabhängige Mitarbeiter,

- Anwendung von Auto-ID Techniken (Strichcode oder RFID Techniken),

- Nachträgliche Analyse von Filmaufnahmen,

- Analyse von Produktionsdokumenten und Statistiken,

- Datenerfassung aus ERP-Systemen

Die obigen Methoden beinhalten diverse Vor- und Nachteile, die je nach Einsatzfall abgewogen werden müssen. Manuelle Aufnahmen durch Mitarbeiter benötigen nur geringe Investitionen und es können damit sehr verschiedene Parameter betrachtet werden. Über einen längeren Zeitraum gesehen ist die manuelle Datenaufnahme aufgrund der laufenden Personalkosten allerdings sehr kostspielig. Um eine langfristige Kostenreduktion bei der Datensammlung zu erreichen, können diese Prozesse automatisiert werden. Hierzu kommen Auto-ID Techniken als sinnvolle Lösung in Frage. Deren Vorteile sind die Anwendung als standardisierte Systembausteine und Schnittstellen. Diese Systeme benötigen allerdings im Vergleich zu den anderen Methoden hohe Investitionskosten, sofern keine Auto-ID Technik vorhanden ist und diese neu beschafft und installiert werden muss. Filmaufnahmen haben den Vorteil, dass die gesammelten visuellen Daten nachträglich analysiert werden können. Die wesentlichen Nachteile dieser Methode können in der Ablenkung der Mitarbeiter und in der lokalen Einschränkung der Datensammlung bestehen. Die nachträgliche Auswertung der Filmaufnahmen beansprucht zusätzlich einen hohen Aufwand. Daten für die Simulation können auch aus verschiedenen Betriebsdokumenten und Statistiken stammen. Deren Einbau in das Modell ist vorteil- haft, da diese Dokumente meistens über eine lange Zeit zur Verfügung stehen. Der Nachteil besteht wieder in der aufwendigen manuellen Datenvorbereitung für die Simulation. Wenn die zur Simulation benötigten Daten schon im ERP-System vorliegen, müssen sie möglichst schon bei der Generierung des Simulationsmodells eingesetzt werden, so dass aufgrund der Zuverlässigkeit der Daten eine automatische Datenkonversion erfolgen kann. Nachteilig kann im Fall der Datenerfassung aus ERP-Systemen sein, wenn die Daten den Ansprüchen der Simulation nicht genügen und somit auch andere Datenquellen benutzt werden müssen.

Aus den beschriebenen Vor- und Nachteilen ist $\mathrm{zu}$ erkennen, dass die verschiedenen Datensammlungsmethoden in unterschiedlichen Anwendungsgebieten zum Einsatz kommen. Das vorgeschlagene Kamerabasierte Datensammlungssystem muss so gestaltet sein, dass es eine sinnvolle Ergänzung zu den bestehenden Datensammlungsmethoden bietet. Das grundsätzliche Arbeitsprinzip des Kamera-basierten Datensammlungssystems lässt sich wie folgt zusammenfassen:

- Die Kamera muss mit verschiedenen Linsen ausrüstbar sein, um an die unterschiedlichsten Aufgaben angepasst werden zu können.

- Das Zeitfenster der Bildaufnahme (shutter speed) muss den Geschwindigkeitsanforderungen des Materialflusssystems genügen.

- Die Struktur der Bearbeitungssoftware soll möglichst allgemein gehalten sein, damit sie für die verschiedenen Anwendungen adaptiert werden kann.

In Kapitel 3 werden die Anwendungsgebiete des Kamera-basierten Datensammlungssystems mit einigen Beispielen verdeutlicht. 


\section{ANWENDUNGSGEBIETE}

Das Kamerasystem für die Datensammlung ist insbesondere für elektromechanische Stückgut-Stetigförderer geeignet - der Einsatz für Schüttgüter und im Bereich pneumatischer oder hydraulischer Stetigförderer ist bisher nicht vorgesehen. Die Anwendung hängt im Wesentlichen von Größe und Anordnung des Materialflusssystems ab.

Die günstigste Anwendung des vorgeschlagenen Kamerasystems liegt im Bereich der Band-, Rollen- und Kreisfördertechnik für standardisierte Ladeeinheiten $(\mathrm{Pa}-$ letten und Boxen). Bei diesen Anlagen fahren die Ladeeinheiten geführt, und so können die einzulernenden Bildausschnitte präziser bestimmt werden. Mit Hilfe eines Kamerasystems können für diese Fördersysteme folgende Betriebsparameter gesammelt werden:

- Zählung und Registrierung der Durchfahrt von Ladeeinheiten an bestimmten Systempunkten

- Zustandserkennung von fördertechnischen Geräten (z. B. Weichen und sonstige Aktoren)

- Verfolgung von Ladeeinheiten durch Weichen

- Zählen der Anzahl der wartenden Ladeeinheiten auf Staustrecken

Bei Fahrerlosen Transportsystemen (FTS) gibt es ähnliche Anwendungsmöglichkeiten. Hierbei kann die Durchfahrt von FTS-Fahrzeugen registriert werden. Kameras zur Datensammlung können auch beim Staplerbetrieb benutzt werden. Die Bestimmung von den $\mathrm{zu}$ analysierenden Bildausschnitten ist in diesem Fall komplizierter, da konventionelle Stapler beim Fahren relativ große Bewegungsfreiheit besitzen. Eine mögliche Lösung dazu liegt in der Ortung des Staplers mit Hilfe einer Bewegungsanalyse und der nachfolgenden Klassifizierung des Bildausschnittes der Bewegung. Bei diesen Systemen kann eine Zustandsanalyse (beladener oder unbeladener Stapler, Stapler mit oder ohne Fahrer) eine zusätzliche Aufgabe sein. Kamerasysteme können darüber hinaus dafür benutzt werden, den Füllstand eines Palettenpuffers über die Zeit aufzunehmen.

Es gibt einige Einsatzfelder, in denen die Systemanwendung der Kamera-basierten Datensammlung aus Platzgründen einige Schwierigkeiten beinhaltet. Der Einsatz in Container-Terminals ist ein Beispiel für die Problematik der Platzierung der Kameras, da in diesem Fall die Sichtfelder beständig angepasst werden müssten und die Anbringung der Kameras schwieriger ist [RPN11]. Fördersysteme für kleine Ladeeinheiten (z. B.: elektronische Komponenten) können ebenfalls problematisch sein, da die Fördertechnik oft mit anderen Anlagenteilen (z. B. Montagegeräte, Roboter, Einheiten zur Qualitätskontrolle) umbaut ist, weshalb für die Platzierung der Kameras nicht ausreichend Platz zur Verfügung stehen kann.
Die obigen Einsatzbeispiele zeigen, dass bewegte Materialflussobjekte mit dieser Methode in vielen Bereichen der Intralogistik identifiziert werden können. Darüber hinaus kann die Erkennung von Objektdetails nur über die Auflösung eines konventionellen Kamerasystems beeinflusst werden. Eine detaillierte Beschreibung der in der Testumgebung eingesetzten Kamera, der Hardware und des entwickelten Programms enthält Kapitel 4.

\section{ARBEITSPRINZIP DER DATENSAMMLUNGSSOFTWARE}

In den vorgestellten Untersuchungen wird eine handelsübliche Internet-Protokoll-Farbkamera (IPFarbkamera) mit PC-Anschluss benutzt. Diese kann Bilder in einem Intervall von 0,3 s speichern. Die entwickelte Bearbeitungssoftware ist sehr flexibel und leicht zu bedienen, damit sie für verschiedene Aufgaben schnell angepasst werden kann. Die grundsätzliche Betriebsweise ist ähnlich der LVQ-Methode (Learning Vector Quantization), einem überwachten Lernverfahren für die Klassifizierung von mehrdimensionalen Vektoren [Koh86]. Bei dieser Methode muss die Kamera vor dem Sammlungsprozess in der entsprechenden Position in der Nähe des untersuchten Systems montiert werden. Diese Position kann während der Datensammlung nicht verändert werden. Wenn eine Kamera nicht ausreicht, müssen mehrere Kameras an das System angeschlossen werden. Nachdem die Kamera fixiert wurde, kann der Sammlungsprozess gestartet werden. Es werden in bestimmten Zeitausschnitten Kamerabilder auf der Festplatte gespeichert. In diesem offline-Betriebsmodus erfolgen Einlernen und Analyse der Bilder zu einem späteren Zeitpunkt. Der Vorteil ist, dass die Entscheidungen des Programms immer auch später nachvollzogen werden können.

Wenn das beobachtete Intervall zu lang ist, wird die zu speichernde Datenmenge sehr groß. Deshalb verfügt das System zusätzlich über einen online-Modus. Hierbei geschieht während der Sammlungsphase nicht nur das Speichern der Bilder, sondern auch die Verarbeitung selbst. Es werden nur die analysierten Daten gespeichert. Diese Methode benötigt im Vorfeld eingelernte Modelle.

Das Einlernen der Modelle beginnt mit der Bestimmung der benötigten Bildbereiche (Area of Interest = AOI). Die Anzahl und Position der auszuwählenden Bildbereiche hängt von der Art des Fördersystems und der gesammelten Daten ab. Beispiele für eingelernte Modelle werden in Kapitel 5 vorgestellt. Im nächsten Schritt müssen für jeden Bereich die benötigen Kategorien, die während der Auswertung und Verarbeitung unterschieden werden sollen, definiert werden. Meistens genügen zwei Kategorien: Objekt im Bereich oder leerer Bereich. Es können auch mehr als zwei Kategorien definiert werden, was notwendig ist, wenn mehrere verschiedenartige Fördereinheiten unterschieden werden müssen. Es ist nicht nötig, dass dieBereiche quadratisch sind, da mit Hilfe von frei $\mathrm{zu}$ definierenden Masken verschiedenste Formen an- 
gegeben werden können. Nach der Bestimmung des Bereichs, der Kategorie und der Maske kann ein Modell mit den zugehörigen drei Parametern (Bereich Nr., Kategorie Nr. und Maske Nr.) definiert werden. Diese Modelle wer- den während des Erkennungsprozesses durch die Software benutzt. Das Arbeitsprinzip des Systems wird in Abbildung 2 veranschaulicht. Während des Erkennungsprozesses kalkuliert die Software den absoluten Abstand des

\begin{tabular}{|l|lll}
\hline \multicolumn{2}{|c|}{ Kamerabild } \\
angetriebene \\
Rollenbahnen
\end{tabular}

Abbildung 2: Arbeitsprinzip des Kamera-basierten Datensammlungssystems

Modells und des aktuellen Bildausschnitts in den $n$ dimensionalen Raum, wobei $n$ der Anzahl der Pixel im Modell entspricht. Dazu wird die folgende Formel in der Software benutzt

$d_{m a}=\sum_{i=1}^{n}\left(a b s\left(r_{m i}-r_{a i}\right)+a b s\left(g_{m i}-g_{a i}\right)+a b s\left(b_{m i}-b_{a i}\right)\right)$

mit

$\mathrm{d}_{\mathrm{ma}}[\mathrm{m}]-$ Kalkulierter Abstand zwischen Pixel eines Modells und zugehörigem Bildbereich

$\mathrm{r}_{\mathrm{mi}}$ [-] - R Komponente der Pixel des Modells

$\mathrm{g}_{\mathrm{mi}}[-]-$ G Komponente der Pixel des Modells

$\mathrm{b}_{\mathrm{mi}}[-]-$ B Komponente der Pixel des Modells

$\mathrm{r}_{\mathrm{ai}}$ [-] - R Komponente der Pixel des aktuellen Bildes

$\mathrm{g}_{\mathrm{ai}}$ [-] - G Komponente der Pixel des aktuellen Bildes

$\mathrm{b}_{\mathrm{ai}}[-]-$ B Komponente der Pixel des aktuellen Bildes

wobei dma dem kalkulierten “Abstand” (Unterschied) zwischen Pixeln eines Modells und des zugehörigen Bildbereichs (AOI) auf dem aktuellen Bild entspricht. Die Größen rmi, gmi und bmi sind RGB-Komponenten der Pixel des Modells und rai, gai und bai sind RGB Komponenten der Pixel des aktuellen Bildes.

Das Ergebnis des Erkennungsprozesses für einen Bildbereich ist die Kategorie, dessen Modell den kleinsten Abstand zu dem aktuellen Bildbereich hat. Das Programm führt den Erkennungsprozess für alle Bildbereiche im aktuellen Bild durch.

\section{ANWENDUNG DER ERGEBNISSE IN EINER SIMULATIONSUMGEBUNG}

Simulationsmodelle liefern häufig entscheidungsrelevante Ergebnisse für die Logistik in Unternehmen. Die Modelle ermöglichen Untersuchungen der Leistungsfähigkeit bestehender Systeme und vorhergehende Wirkungsanalysen von bevorstehenden Systemveränderungen. Die Ergebnisse der Modellexperimente werden im Wesentlichen von den eingegebenen Parameter- und Eingangswerten bestimmt. Mit anderen Worten: Die Verwendung realistischer Daten im Simulationsmodell erhöht die Genauigkeit des Modells und damit auch der Ergebnisse des Modells. Die Daten aus der oben beschriebenen Kamera-basierten Datensammlungsmethode können für verschiedene Simulationszwecke benutzt werden.

Abbildung 3 zeigt eine mögliche Anwendung der Kamera-basierten Datensammlung. Hier wird eine Kamera vor und eine Kamera hinter dem ausgewählten Teil des Fördersystems positioniert. Wenn der Teil des Fördersystems nach dem First-In-First-Out-Prinzip (FIFO-Prinzip) arbeitet und der Zeitpunkt des Eintretens und Verlassens eines ausgewählten Objektes bekannt ist, dann können aus den gesammelten Daten relevante Systemeigenschaften wie die durchschnittliche Durchlaufzeit und Anzahl der Objekte im Systemteil bestimmt werden. Dadurch kann der betrachtete Systemteil im Simulationsmodell mit einem einzigen Element modelliert werden.

Abbildung 4 zeigt die eintretende Situation, wenn nur eine Kamera benutzt wird. Auf diese Weise kann der Materialfluss an einem bestimmten Punkt im System aufgenommen werden. Diese Daten können in solchen Fällen benutzt werden, wenn das nach dem Beobachtungspunkt folgende System modelliert wurde, und vom Anwender gewünscht wird, für dieses System reale Daten einzuge- 
ben. Zusammenfassend kann festgehalten werden, dass Kamerasysteme eine wichtige Überbrückungsfunktion zwischen dem realisierten und dem simulierten Materialflusssystem haben können.

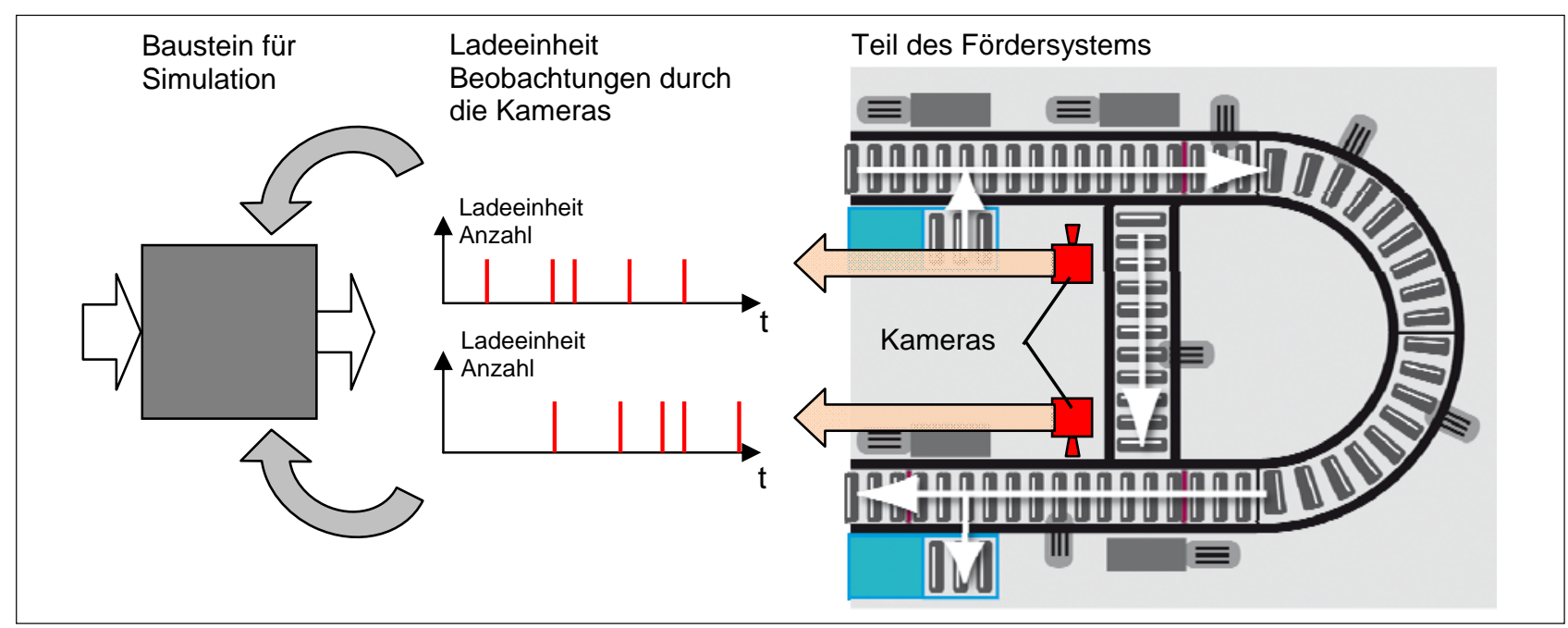

Abbildung 3: „Black box“-Parameteranalyse mit Hilfe von Kameras

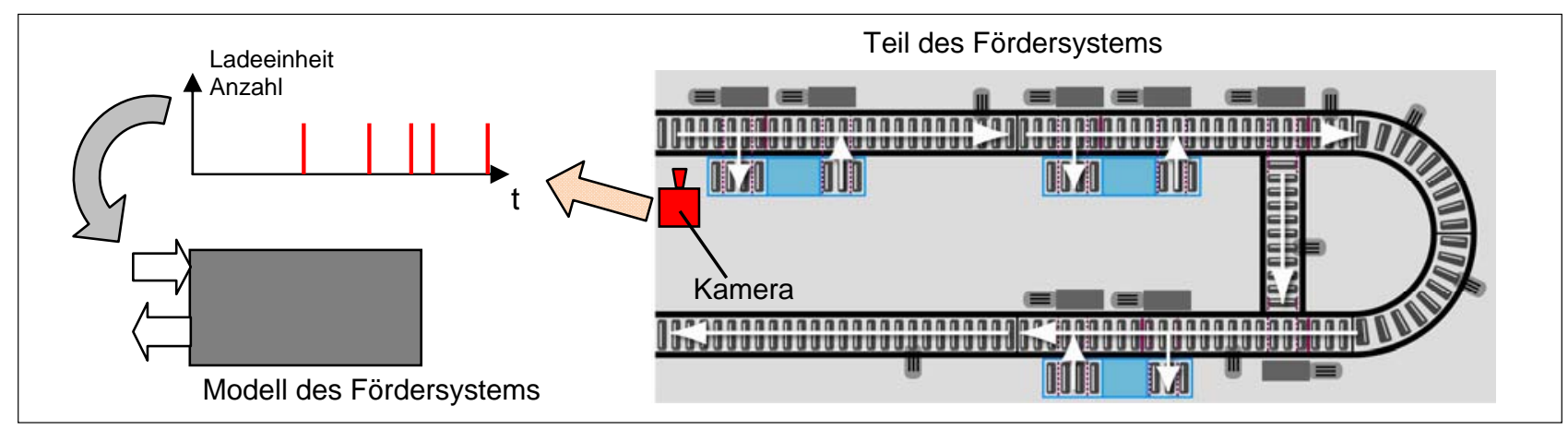

Abbildung 4: Sammlung von Eingabedaten für ein Simulationsmodell 


\section{BEISPIELE FÜR DIE ANWENDUNG}

\subsection{BEISPIEL DREHTISCH}

Der Drehtisch (Abb.5) ist ein einfacher und kompakter Teil eines Rollenbahnsystems. Seine Anwendung ist vorteilhaft, wo ein Richtungswechsel benötigt wird und der Abstand parallel verlaufender Rollenbahnen sehr klein ist. Ladeeinheiten können im Beispiel aus den Richtungen A und B kommen, wobei Richtung A Priorität hat. Wenn sich Ladeeinheiten aus beiden Richtungen gleichzeitig nähern, muss die aus Richtung B kommende Ladeeinheit warten.

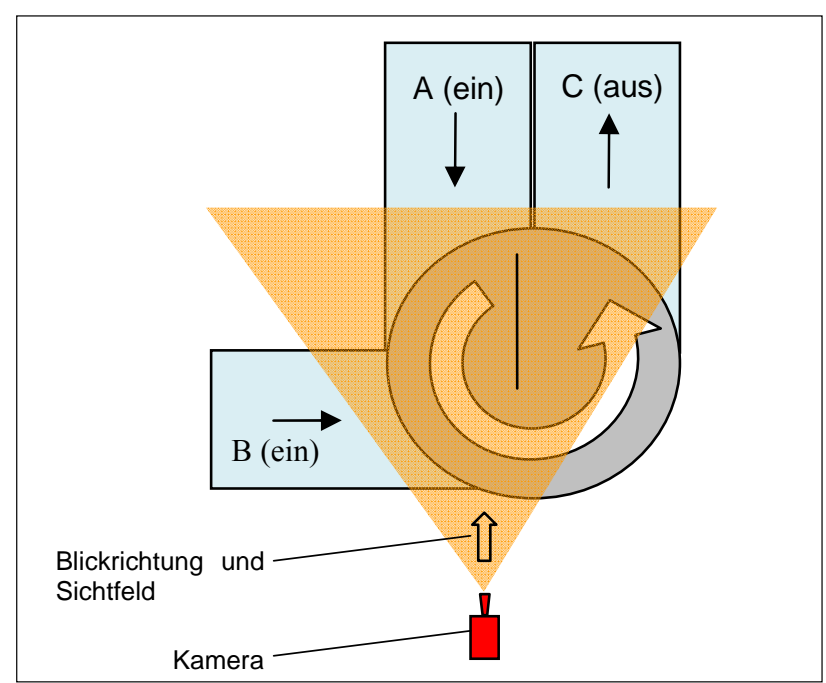

Abbildung 5: Aufbau eines Drehtischs

Das vorgeschlagene Kamerasystem kann für diese Anlage folgende logistische Parameter ausgeben:

- durchschnittliche Wartezeit einer Ladeeinheit am Eingang B,

- zeitliche Auslastung,

- durchschnittlicher Materialflussstrom am Ausgang.

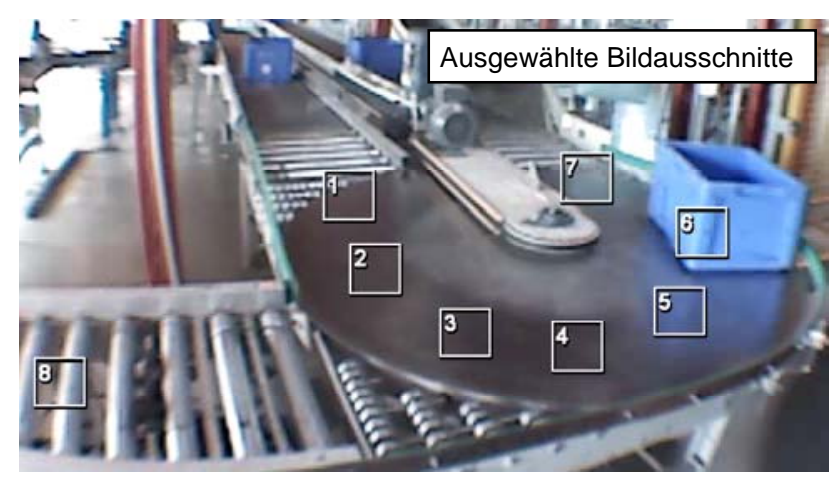

Abbildung 6: Ausgewählte Bildbereiche für einen Drehtisch

Abbildung 6 stellt die gewählten Bildbereiche (AOI) dar. Bereich 1 signalisiert eine ankommende Ladeeinheit aus Richtung A. Die Bereiche 2-7 signalisieren die Belegung des Drehtisches in verschiedenen Positionen. Das bedeutet, dass diese fünf AOI's einen gemeinsamen $\mathrm{Zu}$ stand besitzen: Drehtisch besetzt. AOI 8 signalisiert ankommende oder wartende Ladeeinheiten aus Richtung B. $\mathrm{Zu}$ jedem Bereich gehören zwei Kategorien: Kategorie 1 (Kat.1) bedeutet, dass der Bereich mit der Ladeeinheit belegt ist, Kat. 2 signalisiert einen leeren Bereich.

Während des Einlernprozesses (teach-in Prozess) wurden mehrere Bilder gespeichert und eingespielt. Zwei Schwierigkeiten tauchten während der Erkennung auf: Zum einen spiegelte die Oberfläche des Drehtisches, da sie aus Stahl gefertigt ist. Das Objekt in Abbildung 6 befindet sich im Bereich 6, wobei das blaue Spiegelbild der Box auch im Bereich 5 anstelle der metallischen graue Farbe des Drehtisches zu erkennen ist. Zum anderen existieren Transportbehälter im Beispielsystem mit einem weißen Etikett und schwarzer Beschriftung (s. Abb. 7). Diese Ladeeinheiten mussten noch gesondert eingelernt werden, um alle Boxen einwandfrei erkennen zu können. Während der Testphase genügten 23 Modelle für die ein-

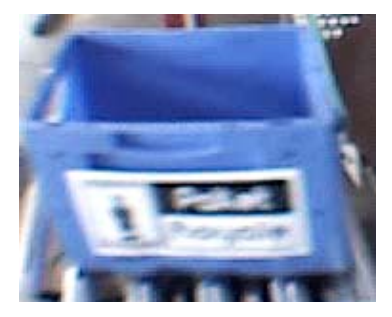
wandfreie Nutzung der Kamera-basierten Stückguterkennung. Dies zeigt, dass die Methode schnell und zuverlässig für diese Anlage adaptiert werden kann.

Abbildung 7:

Ladeeinheit mit Etikett

\subsection{BEISPIEL DREHSORTER}

Der Drehsorter (detaillierte Beschreibung siehe [SS10]) ist ein Sortiersystem mit einer Einschleusung, mehreren Endstellen und einer Sortierleistung von 6.000 Stk./h. In diesem Beispiel wurde die Kamera an einer Endstelle montiert. Die Blickrichtung der Kamera ist in Abbildung 8 durch den eingefärbten Bereich gekennzeichnet.

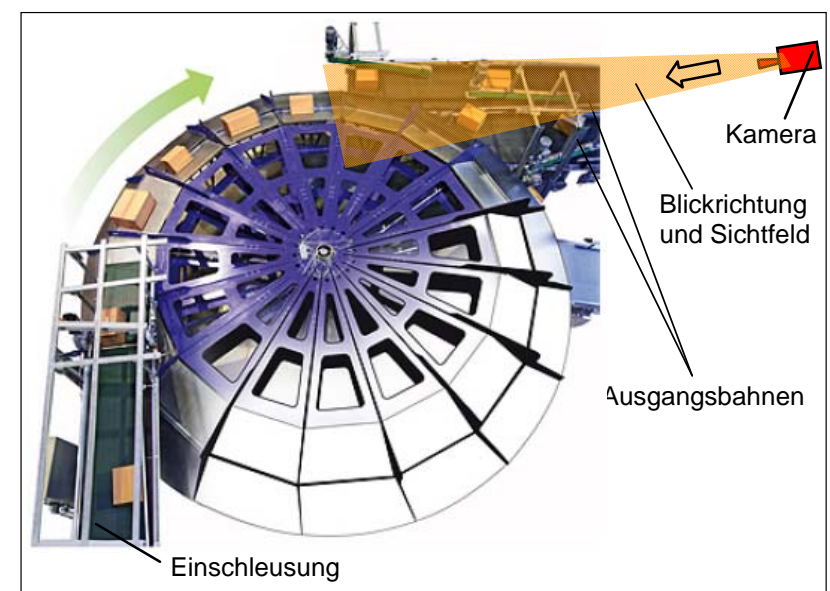

Abbildung 9:

Ausgewählte Bildbereiche für den Drehsorter 
Abbildung 9 stellt die gewählten Bildbereiche dar. Bereich 1 signalisiert die den Drehsorter verlassenden Ladeeinheiten. Zu diesem Bereich gehören zwei Kategorien: Kat. 1 bedeutet, dass der Bereich mit einer Ladeeinheit belegt ist, Kat. 2 stellt den leeren Bereich dar. Bereich 2 signalisiert verfahrende Ladeeinheiten auf dem Bandförderer. $\mathrm{Zu}$ diesem Bereich wird eine weitere Funktionalität hinzugefügt: Im Beispiel soll das Programm Ladeeinheiten, die seitlich ein Etikett besitzen und Ladeeinheiten, die kein Etikett, besitzen voneinander unterscheiden (siehe Abbildung 10). Demnach gehören 3 Kategorien zu Bereich 2: Kat. 1 signalisiert Ladeeinheiten mit weißem Etikett, Kat. 2 bedeutet einen leeren Bereich, Kat. 3 signalisiert Ladeeinheiten ohne Etikett (oder eine auf der Seite liegende Ladeeinheit).

Während der Testphase wurden mehrere Bilder untersucht. Das Einlernen der Modelle in der Testumgebung

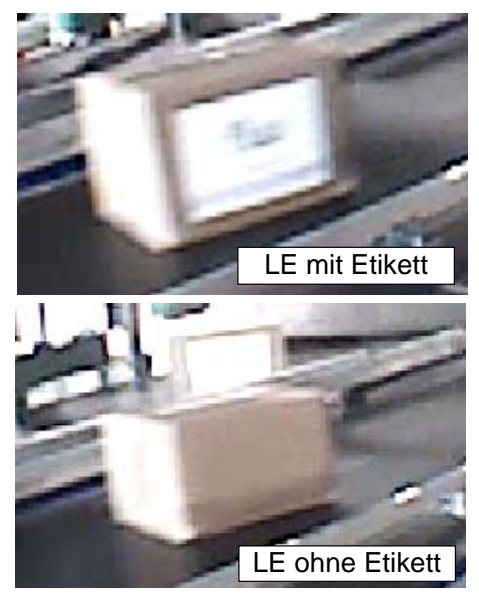
erfolgte ohne Schwierigkeiten und sogar die Drehbewegung des Sorters beeinträchtigte die Erkennungseigenschaften nicht. Schon zehn Modelle reichten für eine einwandfreie Erkennung aus. Daraus wird geschlossen, dass die Methode schnell und zuverlässig auch für diese Anlage adaptiert werden kann.

Abbildung 10

Bilder von Ladeeinheiten

mit und ohne Etikett
Vorgehensweise für mögliche Anwendungen. Die weiteren Schritte der Forschung betreffen die Anwendung der Methode an weiteren Fördersystemen, insbesondere an Flurförderzeugen. Weiterhin ist die Entwicklung der Erkennungskapazität und die Kombination mit anderen Methoden (z.B. Auto-ID Methode) ebenfalls vielversprechend.

\section{LITERATUR}

[Koh86] Kohonen, Teuvo: Learning vector quantization for pattern recognition. Technical Report TKK-F-A601, Helsinki University of Technology, Department of Technical Physics, Laboratory of Computer and Information Science, Finnland, 1986. - ISBN 951-753-950-9

[Pre95] Prezenszky, József: Logisztika (Bevezetö fejezetek). Budapest : Verlag BME Mérnöktovábbképző Intézet, 1995

[RPN11] Richter, Klaus; Poenicke, Olaf; Nykolaychuk, Mykhaylo: Galileo-Testfeld Sachsen-Anhalt: Funk- und bildbasierte Ortung im Hafenbereich. In: Der Kran und sein Umfeld in Industrie und Logistik. [19. Internationale Kranfachtagung, 31. März 2011, Magdeburg], Magdeburg : LOGiSCH, 2011. - ISBN 978-3-930385-74-4, S. $1-8$

[SS10] Semrau, Kai F.; Sadowsky, Volker: Drehsorter neu aufgelegt - bis zu 6.000 Pakete/h eingeschleust und sortiert. In: Tagungsband auf CD zur 1. Central European Conference on Logistics, Miskolc, Ungarn, 2010. ISBN 978-963-661-946-6
Hauptziel dieses Artikels war die Beschreibung einer neuartigen Datensammlungsquelle: ein Kamerasystem mit automatischer Verarbeitungsfunktion. Für den Einsatz werden mögliche Datenquellen für Simulationsmodelle diskutiert und bewertet. Daraus wird der mögliche Anwendungsbereich von Kamerasystemen abgeleitet. Es werden einige experimentelle Erfahrungen mit dem Kamera-basierten Datensammlungssystem präsentiert. Dazu wurde in zwei Testumgebungen die Kamera-basierte Datensammlung erprobt. Die Ergebnisse sind als positiv zu bewerten, da die Methode in einem kurzen Zeitraum und mit einer zuverlässigen Erkennung durchgeführt wurde. Darüber hinaus wurde die Nutzung der gesammelten Daten für Simulationsmodelle beschrieben. Dieser Teil beinhaltet die prinzipielle 